\title{
EGFR is phosphorylated at Ty845 in hepatocellular carcinoma
}

\author{
Rajesh Kannangai, Fikret Sahin and Michael S Torbenson \\ Department of Pathology, The Johns Hopkins University School of Medicine, Baltimore, MD, USA
}

\begin{abstract}
Epidermal growth factor receptor (EGFR) is overexpressed in a significant proportion of hepatocellular carcinomas. Recent studies of EGFR inhibitors to treat hepatocellular carcinoma have been encouraging and better understanding of EGFR signaling may lead to more effective strategies for inhibiting this key pathway. The EGFR can be phosphorylated at different tyrosine sites, leading to subsequent activation of different pathways. Cell line and animal studies have shown that MAPK and STAT-3 are important mediators of the EGFR signal in liver cells. However, little is known about EGFR phosphorylation and subsequent signaling in primary hepatocellular carcinoma. We investigated the site of EGFR phosphorylation by Western blot in 18 hepatocellular carcinomas. Fourteen of 18 hepatocellular carcinomas had detectable EGFR by Western blotting and 13 of 14 showed phosphorylation at tyrosine 845. In contrast, no EGFR phosphorylation was detected at tyrosine 998 , tyrosine 1045, or tyrosine 1068, which signal through other pathways including STAT-3 and MAPK. These findings were further explored by examination of EGFR expression and signaling pathway activation in tissue arrays comprised of 73 hepatocellular carcinomas using antibodies that recognize phosphorylated (or activated) proteins. Tissue array studies also found no correlation between EGFR expression (29\% of cases) and STAT-3 nuclear positivity (16\%), AKT $(4 \%)$, MAPK $(3 \%)$, or STAT-5 $(3 \%)$ positivity, all $P>0.05$. EGFR expression was correlated with hepatitis $B$ infection, but not with tumor size, nuclear grade, or proliferative rate. We conclude that EGFR is phosphorylated at tyrosine 845 in most hepatocellular carcinomas and that EGFR expression by immunohistochemistry does not correlate well with STAT-3, STAT-5, MAPK, or AKT immunostaining.

Modern Pathology (2006) 19, 1456-1461. doi:10.1038/modpathol.3800665; published online 25 August 2006
\end{abstract}

Keywords: epidermal growth factor receptor; hepatocellular carcinoma; fibrolamellar carcinoma; hepatic adenoma

Epidermal growth factor receptor (EGFR) is a member of a proto-oncogene family of receptors important in cell proliferation. EGFR overexpression has been demonstrated in many human carcinomas including breast, stomach, esophageal squamous carcinoma, and hepatocellular carcinoma. ${ }^{1}$ Because of the high prevalence of EGFR overexpression in carcinomas, inhibitors of epidermal growth factor (EGF) signaling are potential therapeutic agents. In hepatocellular carcinomas, overexpression of EGFR has been associated with late-stage disease, ${ }^{2}$ increased cell proliferation, ${ }^{2}$ and the degree of tumor differentiation. ${ }^{2,3}$ Early studies of EGFR inhibitors in hepatocellular carcinoma cell lines ${ }^{4-6}$ and phase II studies in human hepatocellular carcinomas ${ }^{7}$ have been encouraging, and better understanding of the

Correspondence: Dr M Torbenson, MD, Department of Pathology, The Johns Hopkins University School of Medicine, 1503 East Jefferson, Room B314, Bond Street Building, Baltimore, MD 21231, USA.

E-mail: mtorben@jhmi.edu

Received 1 March 2006; revised and accepted 15 June 2006; published online 25 August 2006
EGFR signaling in hepatocellular carcinoma will likely enhance the use of these inhibitors.

In normal hepatocytes, ligand binding to EGFR results in receptor dimerization and activation of several possible pathways that transmit signals to the nucleus including STAT $-1,{ }^{8}$ STAT $-3,{ }^{9}$ STAT- $-5,{ }^{8}$ and MAPK. ${ }^{10}$ EGFR also signals through AKT in some cases. ${ }^{11}$ EGFR can be phosphorylated at multiple sites and the site of phosphorylation correlates with the subsequently activated signaling pathway. EGFR signals through STAT-1, STAT-3, and STAT-5 along with MAPK $^{10}$ in non-neoplastic hepatocytes, but the signaling routes are largely unknown in hepatocellular carcinoma. Thus, we determined the site of EGFR phosphorylation in primary hepatocellular carcinoma and also examined key signaling pathways potentially activated by EGFR.

\section{Materials and methods}

Tissue Arrays

Tissue arrays were constructed from formalin-fixed, paraffin-embedded tissues using 73 hepatocellular 
carcinomas, 13 fibrolamellar carcinomas, and 15 hepatic adenomas. Each case was represented by at least four $1.5 \mathrm{~mm}$ cores of neoplastic and paired non-neoplastic liver tissues. In addition, four cases were included on the arrays twice on two different blocks to serve as internal controls for reproducibility of staining.

Five micron sections from paraffin-embedded tissue arrays were immunostained following heat antigen retrieval. The STAT-3, STAT-5, MAPK, and AKT antibodies recognize only phosphorylated (or activated) protein, allowing separation from pools of inactive proteins. The distribution of staining of the neoplastic cells was scored as negative (0), 1-25\% $(1+), 26-50 \%(2+), 51-75 \%(3+)$, and greater than $75 \%(4+)$. Intensity of staining was scored on a scale of $0-3+$.

EGFR immunostain results were also correlated with tumor size and nuclear grade using the modified Edmondson nuclear grade, which has a scale of 1-4. Cell proliferation was studied using Ki-67 and 200 neoplastic cells were counted to determine the percent of positive nuclei.

\section{Cell Lines}

HepG2, Hep3B, and A431 cell lines were purchased from ATCC and propagated in minimum essential medium (Eagle) as per ATCC instructions. HepG2 served as a negative control for EGFR expression, ${ }^{10}$ whereas Hep3B and A431 served as positive controls. EGF was obtained from Invitrogen (Carlsbad, CA, USA) and used at a concentration of $100 \mathrm{ng} / \mathrm{ml}$. Cells were harvested at baseline and 20 min after EGF exposure.

\section{Protein Expression in Cell Lines and Primary Liver Tumors by Western Blot}

After obtaining appropriate Institutional Review Board approval, fresh tissues were collected at the time of surgery from primary liver neoplasms and adjacent non-neoplastic liver tissues. These tissues were different from the archival tissues used to construct the tissue arrays. Tissue was harvested, snap frozen in liquid nitrogen, and stored at $-80^{\circ} \mathrm{C}$ before use. Each case had paired non-neoplastic liver tissues. The tissue diagnoses were confirmed in all cases by routine light microscopy.

Total protein was extracted as per the manufacturer's instructions using the M-PER mammalian protein extraction reagent (Pierce, Rockford, IL, USA). Protein concentrations were demonstrated by a dye-binding assay according to the manufacturer's instructions (Pierce Chemical Co, Rockford, IL, USA). For immunoblot assays, liver proteins (60 $\mu \mathrm{g} /$ lane) in Lamelli buffer were separated by polyacrylamide gel electrophoresis and transferred to nylon membranes. A brief incubation in 5\% lowfat milk was used to block nonspecific binding and
Table 1 Antibodies used for immunohistochemistry and Western blotting

\begin{tabular}{lcc}
\hline Antibody & $\begin{array}{c}\text { Immunohistochemistry } \\
\text { dilution }\end{array}$ & $\begin{array}{c}\text { Western blot } \\
\text { dilution }\end{array}$ \\
\hline Total EGFR & $1: 100$ & $1: 500$ \\
pEGFR (Tyr845) & $1: 20$ & $1: 500$ \\
pEGFR (Tyr992) & NA & $1: 500$ \\
pEGFR (Tyr1045) & NA & $1: 500$ \\
pEGFR (Tyr1068) & NA & $1: 500$ \\
pSTAT-3 (Tyr705) & $1: 10$ & $1: 500$ \\
pSTAT-5 (Tyr694) & $1: 20$ & $1: 500$ \\
p44/42 MAPK & $1: 50$ & $1: 500$ \\
(Thr202/Tyr204) & $1: 100$ & $1: 500$ \\
Total AKT & $1: 100$ & $1: 500$ \\
PAKT & & \\
\hline
\end{tabular}

$\mathrm{p}$ in the given proteins refers to phosphorylated.

membranes were incubated for $4 \mathrm{~h}$, washed, and exposed to secondary antiserum.

\section{Antibodies}

Primary antibodies were all from Cell Signaling Technology (Beverly, MA, USA) and were used according to the manufacturer's directions (Table 1).

\section{Results}

\section{Demographics}

The hepatocellular carcinomas on the tissue arrays were from 54 male patients and 19 female patients, with an average age of $54 \pm 18$ years, range 10-85 years. In 44 cases, clinical information regarding the underlying liver diseases was known: hepatitis C $(N=17)$, hepatitis B (seven), hepatitis C and B coinfection (two), ethanol cirrhosis (five), nonalcoholic fatty liver disease (one), primary sclerosing cholangitis (one), autoimmune cirrhosis (one), cryptogenic cirrhosis or no known liver disease (10). The average tumor size was $6.4 \pm 4 \mathrm{~cm}$; median $5.0 \mathrm{~cm}$. The fibrolamellar carcinomas were from 10 female patients and three male patients with a median age of 27 years. Nine of the fibrolamellar carcinomas were primary tumors, with an average size of $9.7 \pm 3 \mathrm{~cm}$, whereas the remaining tumors were from metastatic tumor deposits in lungs or lymph nodes. The 15 hepatic adenomas were all in women with histories of excess estrogen exposure but no other liver disease. ${ }^{12}$

For the Western blot studies, the hepatocellular carcinomas were from 18 persons, 12 men and six women, with a mean age of $58 \pm 1$ years (Table 2). The average tumor size was $4.8 \pm 3 \mathrm{~cm}$ and all were typical hepatocellular carcinomas. The underlying liver diseases for those with cirrhosis $(N=12)$ were mostly chronic hepatitis C infection $(N=6)$ or cryptogenic cirrhosis $(N=4)$. Six individuals had 
Table 2 Immunostaining results

\begin{tabular}{lcccc}
\hline Immunostain & $\begin{array}{c}\text { Total } \\
\text { positive }\end{array}$ & $\begin{array}{c}\text { Hepatocellular } \\
\text { carcinoma, N=73 }\end{array}$ & $\begin{array}{c}\text { Fibrolamellar } \\
\text { carcinoma, N=13 }\end{array}$ & $\begin{array}{c}\text { Hepatic adenoma, } \\
\mathrm{N}=15\end{array}$ \\
\hline Total EGFR & 32 & $21(29 \%)$ & $7(54 \%)$ & $4(22 \%)$ \\
Phospho-STAT-3 & 16 & $12(16 \%)$ & $4(31 \%)$ & 0 \\
Phospho-STAT-5 & 3 & $2(3 \%)$ & 0 & $1(7 \%)$ \\
Phospho-MAPK (ERK1/2) & 2 & $2(3 \%)$ & $1(8 \%)$ & 0 \\
Total AKT & 11 & $10(14 \%)$ & $1(8 \%)$ & 0 \\
Phospho-AKT & 4 & $3(4 \%)$ & 0 \\
\hline
\end{tabular}

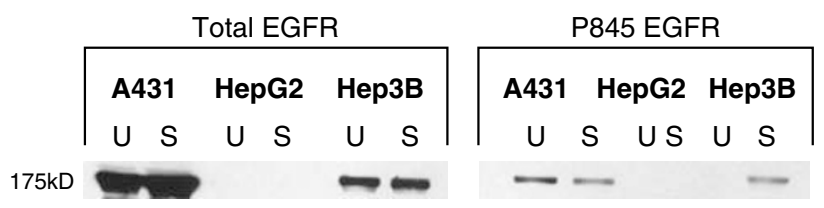

Figure 1 A431 and Hep3B cell lines are positive for total EGFR both before and after stimulation by EGF. HepG2 is negative. Phosphorylation at Tyr-845 is seen in A431 cells before and after stimulation, whereas phosphorylation at this site is seen in Hep3B only after EGF stimulation.

no fibrosis or only mild liver fibrosis at the time of partial hepatectomy.

\section{Western Blot Findings}

Hep3B was positive for EGFR expression as was A431 (positive controls), whereas HepG2 was negative (negative control) (Figure 1). Following EGF stimulation, Hep3B showed expression of total EGFR and demonstrated phosphorylation at Tyr845 (Figure 1). HepG2 was negative for EGFR both before and after EGF stimulation, whereas A431 cells, which are known to overexpress EGFR, were positive for all phosphorylation sites after stimulation.

In the patient samples, EGFR expression was detectable in $8 / 18(44 \%)$ of non-neoplastic livers and in 14/18 (78\%) of hepatocellular carcinomas (Figure 2a; Table 3). Additional Western blots using antibodies to different phosphorylation sites on EGFR found phosphorylation only at Tyr845 (Figure 2b).

\section{Immunostaining Results}

By immunohistochemistry, total EGFR was overexpressed in $21 / 73$ (29\%) of typical hepatocellular carcinomas and $7 / 13(54 \%)$ of fibrolamellar carcinomas (Table 2). EGFR staining showed a membranous pattern in all cases with no cytoplasmic or nuclear positivity (Figure 3a). The non-neoplastic tissues were negative with rare exception when focal positivity was present. The intensity of staining in the hepatocellular carcinomas and fibro-

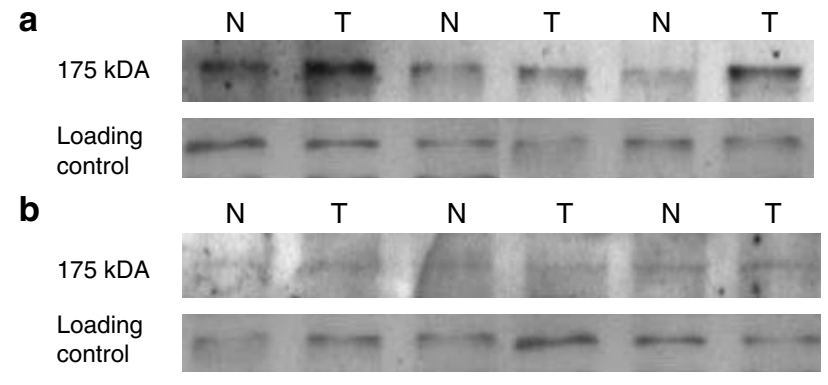

Figure 2 (a) Western blot analysis for EGFR of three typical cases of hepatocellular carcinoma with paired non-neoplastic tissues. (b) EGFR is overexpressed in the first and third case. Phosphorylation at Ty-845 is also detectable.

lamellar carcinomas was $1+(N=18$ cases $), 2+(6)$, and $3+(4)$. All but one of the cases showed diffuse distribution $(3+$ to $4+)$. Interestingly, EGFR positivity was more common in cases with hepatitis $\mathrm{B}$ viral infection, $P=0.029$. However, EGFR positivity did not correlate with nuclear grade, Ki-67 labeling index, or tumor size, $P=0.4,0.3$, and 0.6 , respectively.

Additional staining for phospho-STAT3, phospho-STAT5, phospho-MAPK, and for total and phospho-AKT showed variable positivity in hepatocellular carcinomas and fibrolamellar carcinomas, but no correlation with EGFR positivity, all $P>0.05$. Phospho-STAT-3 nuclear accumulation (Figure 3b) was most common and was present in 12/73 (16\%) of hepatocellular carcinomas and 4/13 (31\%) of fibrolamellar carcinomas. Phospho-STAT-5 showed nuclear positivity in 2/73 hepatocellular carcinomas $(3 \%)$. One hepatocellular carcinoma showed overexpression of cytoplasmic MAPK and a single, separate case showed nuclear accumulation. Total AKT was overexpressed in 10/73 hepatocellular carcinomas (14\%) and one fibrolamellar carcinomas (8\%), whereas phospho-AKT was found in $3 / 10$ hepatocellular carcinomas and the single fibrolamellar carcinoma that showed total AKT overexpression.

Four $(22 \%)$ hepatic adenomas were EGFR positive and one of these also showed overexpression of cytoplasmic phospho-STAT5. 
Table 3 Western blot results for EGFR

\begin{tabular}{|c|c|c|c|c|c|c|c|c|c|c|}
\hline Case no. & Age/sex & Surgery & $\begin{array}{l}\text { Underlying } \\
\text { liver } \\
\text { disease }\end{array}$ & $\begin{array}{l}\text { Fibrosis } \\
\text { stage }^{\mathrm{a}}\end{array}$ & $\begin{array}{c}\text { Tumor } \\
\text { size }(\mathrm{cm})\end{array}$ & $\begin{array}{c}E G F R \\
\text { total } N / T^{\mathrm{b}}\end{array}$ & $\begin{array}{c}\text { EGFR } \\
\text { Tyr845 N/T }\end{array}$ & $\begin{array}{c}\text { EGFR } \\
\text { Tyr998 N/T }\end{array}$ & $\begin{array}{c}\text { EGFR } \\
\text { Tyr1045 N/T }\end{array}$ & $\begin{array}{c}\text { EGFR } \\
\text { Tyr1068 N/T }\end{array}$ \\
\hline 1 & $40 / \mathrm{M}$ & Transplant & $\mathrm{HCV}$ & 6 & 5.5 & $-1-$ & $-1-$ & $-1-$ & $-1-$ & $-1-$ \\
\hline 2 & $67 / \mathrm{M}$ & Transplant & HCV & 6 & 4 & $+/+$ & $-1-$ & $-1-$ & $-1-$ & $-1-$ \\
\hline 3 & $65 / \mathrm{M}$ & Transplant & $\mathrm{HCV}$ & 6 & 1 & $+/+$ & $+/+$ & $-1-$ & $-1-$ & $-1-$ \\
\hline 4 & $61 / \mathrm{F}$ & Transplant & $\mathrm{HCV}$ & 6 & 1 & $+/+$ & $+/+$ & $-1-$ & $-1-$ & $-1-$ \\
\hline 5 & $61 / \mathrm{M}$ & Resection & $\mathrm{HCV}$ & 6 & 4.4 & $-/+$ & $-1+$ & $-1-$ & $-1-$ & $-1-$ \\
\hline 6 & $66 / \mathrm{M}$ & Resection & $\mathrm{HCV}$ & 1 & 4.7 & $-1-$ & $-1-$ & $-1-$ & $-1-$ & $-1-$ \\
\hline 7 & $59 / F$ & Resection & $\mathrm{HBV}$ & 6 & 2.5 & $+/+$ & $+/+$ & $-1-$ & $-1-$ & $-1-$ \\
\hline 8 & $60 / \mathrm{M}$ & Transplant & Cryptogenic & 6 & 2 & $+/+$ & $+/+$ & $-1-$ & $-1-$ & $-1-$ \\
\hline 9 & $54 / \mathrm{M}$ & Transplant & Cryptogenic & 6 & 1.5 & $+1-$ & $+1-$ & $-1-$ & $-1-$ & $-1-$ \\
\hline 10 & $54 / \mathrm{F}$ & Transplant & Cryptogenic & 6 & 4 & $-1+$ & $-1+$ & $-1-$ & $-1-$ & $-1-$ \\
\hline 11 & $53 / \mathrm{M}$ & Transplant & Cryptogenic & 6 & 3.5 & $+/+$ & $+/+$ & $-1-$ & $-1-$ & $-1-$ \\
\hline 12 & $63 / \mathrm{M}$ & Resection & ETOH & 6 & 5 & $-/+$ & $-/+$ & $-1-$ & $-1-$ & $-1-$ \\
\hline 13 & $70 / \mathrm{M}$ & Resection & None & 1 & 7.5 & $-1+$ & $-1+$ & $-1-$ & $-1-$ & $-1-$ \\
\hline 14 & $70 / \mathrm{M}$ & Resection & None & 0 & 6.5 & $-1+$ & $-1+$ & $-1-$ & $-1-$ & $-1-$ \\
\hline 15 & $25 / F$ & Resection & None & 0 & 2.5 & $-1+$ & $-1+$ & $-1-$ & $-1-$ & $-1-$ \\
\hline 16 & $40 / \mathrm{F}$ & Resection & None & 0 & 3.7 & $-1+$ & $-1+$ & $-1-$ & $-1-$ & $-1-$ \\
\hline 17 & $81 / \mathrm{M}$ & Resection & None & 0 & 8 & $+/+$ & $+/+$ & $-1-$ & $-1-$ & $-1-$ \\
\hline 18 & $36 / \mathrm{F}$ & Resection & None & 0 & 12 & $-1-$ & $-1-$ & $-1-$ & $-1-$ & $-1-$ \\
\hline \multicolumn{11}{|l|}{ Cell lines } \\
\hline $\mathrm{HepG}^{\mathrm{c}}$ & NA & NA & NA & NA & NA & - & - & - & - & - \\
\hline HepG $2^{\mathrm{d}}$ & & & & & & - & - & - & - & - \\
\hline Hep3B $^{\mathrm{c}}$ & NA & NA & NA & NA & NA & + & - & - & - & - \\
\hline${\text { Hep} 3 B^{d}}^{d}$ & & & & & & + & + & - & - & + \\
\hline $\mathrm{A} 431^{\mathrm{c}}$ & NA & NA & NA & NA & NA & + & + & - & - & - \\
\hline $\mathrm{A} 431^{\mathrm{d}}$ & & & & & & + & + & + & + & + \\
\hline
\end{tabular}

${ }^{\mathrm{a}}$ The fibrosis is staged from 0 (no fibrosis) to 6 (cirrhosis).

${ }^{\mathrm{b}} N=$ non-neoplastic liver tissue; $\mathrm{T}=$ tumor.

${ }^{\mathrm{c}}$ before EGF stimulation.

$\mathrm{d}_{\text {after EGF stimulation. }}$

\section{Discussion}

EGFR is an attractive candidate for therapeutic intervention and several inhibitors have been developed. In this study, we have demonstrated that EGFR is detectable by immunohistochemistry in $29 \%$ of hepatocellular carcinomas, $54 \%$ of fibrolamellar carcinomas, and $22 \%$ of hepatic adenomas. These findings underscore the importance of EGFR signaling in a wide variety of primary hepatic neoplasms. EGFR expression in a high proportion of fibrolamellar carcinomas has also recently been described by another group. ${ }^{13}$ This is the first report of EGFR expression in hepatic adenomas to our knowledge. We have further extended our understanding of EGFR expression in primary hepatocellular carcinoma by showing that only a small proportion of EGFR-positive cases demonstrate activation of AKT or MAPK by immunohistochemistry, suggesting that the pathways are not activated.

EGFR signaling following ligand binding is complex and incompletely understood, but the signal is transduced via different pathways leading to broadly different cellular responses. For example, EGFR signaling through MAPK is mitogenic but inhibitory to production of acute phase proteins, whereas EGFR signaling through STAT-3 stimulates production of acute phase proteins. ${ }^{10}$ Because of its role in cell cycling of normal hepatocytes, EGFR signaling through the MAPK pathway in hepatocellular carcinomas would have been anticipated. However, we found no correlation between EGFR overexpression and pMAPK positivity, along with no correlation with phosphorylation of STAT-3, STAT-5, or AKT. These data raise the possibility that EGFR signaling in primary hepatocellular carcinomas may utilize other signaling pathways. Supporting this notion, EGFR phosphorylation was not evident at Tyr998, Tyr1045, or Tyr1068, phosphorylation sites linked to activation of MAPK, AKT, and STAT signaling pathways. Instead, phosphorylation was found at tyrosine 845 , a location that has been linked to src activity. ${ }^{14}$ These results highlight the need for more studies to understand the mechanisms of EGFR signaling in primary hepatocellular carcinoma.

The signaling pathway activated by EGFR appears to have a profound impact on the sensitivity of tumors to EGFR inhibition. For example, in a clinical treatment study of non-small-cell lung cancer patients, gefitinib's action was dependent on EGFR activating the AKT pathway, but not the MAPK pathway. ${ }^{15}$ Likewise, in hepatocellular carcinoma cell lines, the effect of gefitinib was pathway 
1460

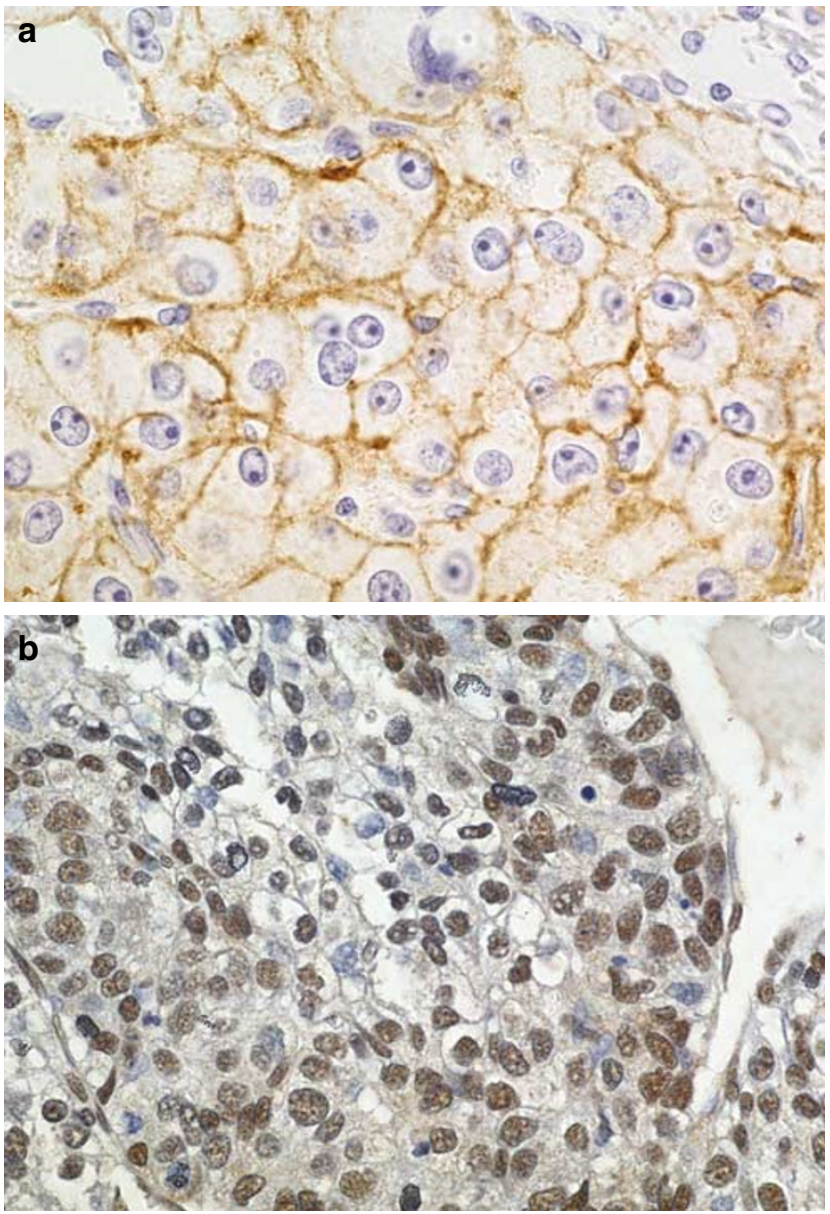

Figure 3 (a) EGFR shows strong membranous staining in a typical case of hepatocellular carcinoma. (b) Phospho-STAT-3 nuclear labeling in a typical case of hepatocellular carcinoma.

dependent. ${ }^{5,16}$ In fact, the effect of EGFR inhibition by gefitinib was duplicated by individually blocking the downstream AKT and MAPK pathways. In contrast, those EGFR-positive cell lines that did not activate these pathways were not inhibited. ${ }^{16}$ Thus, the observation that EGFR protein expression per se does not predict efficacy of EGFR inhibition $^{6,17-19}$ is likely explained by the fact that efficacy is more dependent on the subsequent signaling pathway and less on the quantitative expression of EGFR and has led to the proposal that testing for other markers, such as pAKT, may be needed to select patients for EGFR inhibitor therapy. ${ }^{15,19-21}$ This point is further emphasized by the recent report of a phase II clinical trial of Erlotinib in patients with hepatocellular carcinoma. ${ }^{7}$ Phillip et al found that $3 / 38$ patients showed a partial radiological response and 12/38 were progression free at 6 months. Although these results are very encouraging, the response rate is not well explained by EGFR expression in the tumors, which was present in nearly all (92\%) cases. Interestingly, Phillip et $a l^{7}$ also reported that patients with hepatitis C-related hepatocellular carcinoma had the best response to therapy and also noted that hepatitis $\mathrm{C}$ has been associated with MAPK activation by others, suggesting that signaling pathway activation may be the feature that best defines response to therapy.

In this study, a much higher proportion of hepatocellular carcinomas were EGFR positive by Western blotting than by immunostaining. This discrepancy likely represents differences in sensitivity between methods, but a direct comparison was not possible as the same cases were not used for both immunostaining and Western blotting. An additional limit to our findings is that the cell culture studies are based on EGFR signaling following EGF stimulation. However, other ligands, such as transforming growth factor alpha, also bind to EGFR and it is unclear if our findings can be extended to stimulation by ligands other than EGF.

An additional limit to understanding EGFR overexpression in hepatocellular carcinoma is the diverse number of protocols used to detect EGFR, a point underscored by the variable immunopositivity for EGFR in the non-neoplastic liver tissues, ranging from $15 \%^{2}$ to none in this study. Nevertheless, focusing only on those studies in which EGFR expression in hepatocellular carcinoma was detected by immunohistochemistry, the frequency of positivity ranges from 3 to $85 \% .^{2,22-24}$ The wide range of positivity by immunohistochemistry appears to be explained in part by the underlying causes of liver disease, as hepatitis B virus infection was associated with EGFR overexpression in this and other studies. ${ }^{25,26}$

In conclusion, $29 \%$ of hepatocellular carcinomas in this series showed expression of EGFR by immunohistochemistry. Immunostaining for potential downstream targets of EGFR, including STAT-3, AKT, and MAPK, did not correlate with EGFR expression, suggesting that other pathways may be important in EGFR signaling in hepatocellular carcinoma. Correlating with these findings, the EGFR receptor was phosphorylated preferentially at tyrosine 845 in hepatocellular carcinomas and not at other locations that have been linked to AKT and MAPK signaling.

\section{References}

1 Normanno N, Maiello MR, De Luca A. Epidermal growth factor receptor tyrosine kinase inhibitors (EGFR-TKIs): simple drugs with a complex mechanism of action? J Cell Physiol 2003;194:13-19.

2 Ito Y, Takeda T, Sakon M, et al. Expression and clinical significance of erb-B receptor family in hepatocellular carcinoma. Br J Cancer 2001;84:1377-1383.

3 Kira S, Nakanishi T, Suemori S, et al. Expression of transforming growth factor alpha and epidermal growth factor receptor in human hepatocellular carcinoma. Liver 1997;17:177-182. 
4 Giannelli G, Azzariti A, Sgarra C, et al. ZD6474 inhibits proliferation and invasion of human hepatocellular carcinoma cells. Biochem Pharmacol 2006;71:479-485.

5 Hopfner M, Sutter AP, Huether A, et al. Targeting the epidermal growth factor receptor by gefitinib for treatment of hepatocellular carcinoma. J Hepatol 2004; 41:1008-1016.

6 Huether A, Hopfner M, Baradari V, et al. EGFR blockade by cetuximab alone or as combination therapy for growth control of hepatocellular cancer. Biochem Pharmacol 2005;70:1568-1578.

7 Philip PA, Mahoney MR, Allmer C, et al. Phase II study of Erlotinib (OSI-774) in patients with advanced hepatocellular cancer. J Clin Oncol 2005;23: 6657-6663.

8 Runge DM, Runge D, Foth $\mathrm{H}$, et al. STAT 1alpha/1beta, STAT 3 and STAT 5: expression and association with cMET and EGF-receptor in long-term cultures of human hepatocytes. Biochem Biophys Res Commun 1999; 265:376-381.

9 Runge DM, Runge D, Dorko K, et al. Epidermal growth factor- and hepatocyte growth factor-receptor activity in serum-free cultures of human hepatocytes. J Hepatol 1999;30:265-274.

10 Wang Y, Ripperger J, Fey GH, et al. Modulation of hepatic acute phase gene expression by epidermal growth factor and Src protein tyrosine kinases in murine and human hepatic cells. Hepatology 1999;30: 682-697.

11 Jorissen RN, Walker F, Pouliot N, et al. Epidermal growth factor receptor: mechanisms of activation and signalling. Exp Cell Res 2003;284:31-53.

12 Torbenson M, Lee JH, Choti M, et al. Hepatic adenomas: analysis of sex steroid receptor status and the Wnt signaling pathway. Mod Pathol 2002;15: 189-196.

13 Buckley AF, Burgart LJ, Kakar S. Epidermal growth factor receptor expression and gene copy number in fibrolamellar hepatocellular carcinoma. Hum Pathol 2006;37:410-414.

14 Biscardi JS, Maa MC, Tice DA, et al. c-Src-mediated phosphorylation of the epidermal growth factor receptor on Tyr845 and Tyr1101 is associated with modulation of receptor function. J Biol Chem 1999; 274:8335-8343.

15 Cappuzzo F, Magrini E, Ceresoli GL, et al. Akt phosphorylation and gefitinib efficacy in patients with advanced non-small-cell lung cancer. J Natl Cancer Inst 2004;96:1133-1141.

16 Okano J, Matsumoto K, Nagahara T, et al. Gefitinib and the modulation of the signaling pathways downstream of epidermal growth factor receptor in human liver cancer cells. J Gastroenterol 2006;41:166-176.

17 Sirotnak FM, Zakowski MF, Miller VA, et al. Efficacy of cytotoxic agents against human tumor xenografts is markedly enhanced by coadministration of ZD1839 (Iressa), an inhibitor of EGFR tyrosine kinase. Clin Cancer Res 2000;6:4885-4892.

18 Baguley BC, Marshall ES, Holdaway KM, et al. Inhibition of growth of primary human tumour cell cultures by a 4-anilinoquinazoline inhibitor of the epidermal growth factor receptor family of tyrosine kinases. Eur J Cancer 1998;34:1086-1090.

19 Cappuzzo F, Gregorc V, Rossi E, et al. Gefitinib in pretreated non-small-cell lung cancer (NSCLC): analysis of efficacy and correlation with HER2 and epidermal growth factor receptor expression in locally advanced or metastatic NSCLC. J Clin Oncol 2003; 21:2658-2663.

20 Ono M, Hirata A, Kometani T, et al. Sensitivity to gefitinib (Iressa, ZD1839) in non-small cell lung cancer cell lines correlates with dependence on the epidermal growth factor (EGF) receptor/extracellular signal-regulated kinase 1/2 and EGF receptor/Akt pathway for proliferation. Mol Cancer Ther 2004;3:465-472.

21 de Braud F, De Pas T, Spaggiari L, et al. Re: Akt phosphorylation and gefitinib efficacy in patients with advanced non-small-cell lung cancer. J Natl Cancer Inst 2005;97:461-462; author reply 2-3.

22 Altimari A, Fiorentino M, Gabusi E, et al. Investigation of ErbB1 and ErbB2 expression for therapeutic targeting in primary liver tumours. Dig Liver Dis 2003;35: 332-338.

23 Nakopoulou L, Stefanaki K, Filaktopoulos D, et al. C-erb-B-2 oncoprotein and epidermal growth factor receptor in human hepatocellular carcinoma: an immunohistochemical study. Histol Histopathol 1994; 9:677-682.

24 Yamaguchi K, Carr BI, Nalesnik MA. Concomitant and isolated expression of TGF-alpha and EGF-R in human hepatoma cells supports the hypothesis of autocrine, paracrine, and endocrine growth of human hepatoma. J Surg Oncol 1995;58:240-245.

25 Menzo S, Clementi M, Alfani E, et al. Trans-activation of epidermal growth factor receptor gene by the hepatitis B virus X-gene product. Virology 1993; 196:878-882.

26 Miyaki M, Sato C, Sakai K, et al. Malignant transformation and EGFR activation of immortalized mouse liver epithelial cells caused by HBV enhancer-X from a human hepatocellular carcinoma. Int J Cancer 2000; 85:518-522. 